\title{
Mapping the epileptic brain with EEG dynamical connectivity: established methods and novel approaches
}

\author{
Margarita Papadopoulou and Daniele Marinazzo \\ Department of Data Analysis, Faculty of Psychology and Pedagogical Sciences, \\ Ghent University, Henri Dunantlaan 1, B-9000 Ghent, Belgium \\ Kristl Vonck and Paul Boon \\ Laboratory for Clinical and Experimental Neurophysiology, \\ Ghent University Hospital, B-9000 Ghent, Belgium
}

\begin{abstract}
Several algorithms rooted in statistical physics, mathematics and machine learning are used to analyze neuroimaging data from patients affected by epilepsy, with the main goals of localizing the brain region where seizure originates from and of detecting upcoming seizure activity in order to trigger therapeutic neurostimulation devices. Some of these methods explore the dynamical connections between brain regions, exploiting the high temporal resolution of the electroencephalographic signals recorded at the scalp or directly from the cortical surface or in deeper brain areas. In this paper we describe this specific class of algorithms and their clinical application, by reviewing the state of the art and reporting their application on EEG data from an epileptic patient.
\end{abstract}




\section{Introduction}

Epilepsy is a common brain disorder with various etiologies, affecting roughly 50 million people worldwide. In many cases epileptic seizures can be controlled by antiepileptic drugs, which are nonetheless ineffective in about one third of the patients [1]. For these patients more invasive treatments are available: surgical removal of the epileptogenic region or implantation with a neurostimulation device [2]. Advanced techniques for data analysis can be of great help to optimize the success rate of both therapies, by improving epileptologists' interpretation of complex electroencephalogram (EEG) signals and by maximizing the correct and timely detection of an upcoming seizure.

Epilepsy involves recurrent seizures which are characterized by an increase in accumulated energy in specific frequency bands and brain regions. The rapid seizure propagation and its unpredictable nature render the localization of the epileptic focus and the study of its propagation a challenging task. In order to gather information about a physiological system one can measure the temporal evolution of one or more signals which are reflecting its activity. Concerning epilepsy, this has historically been accomplished by the analysis of the EEG recorded from the scalp or from implanted intracranial electrodes (iEEG). The need to quantify the interactions between different brain regions at the same time, when for example large areas of cortex are in synchronous activity, has led to an extensive development and use of multivariate time series techniques. These techniques can be used to detect patterns of interactions between different brain areas and to improve the understanding of the neural information transfer.

Epileptic seizures evolve dynamically thereby modulating local and distributed neuronal networks. Thus, theories and algorithms developed, validated and optimized in the framework of the analysis of dynamic connectivity may provide valuable tools to elucidate the mechanisms underlying epileptic seizures. A crucial question to answer then, is how the epileptiform activity is related to the connectivity of a network of brain regions and how this network topology changes in function of different states (interictal, preictal, ictal) that occur in the epileptic brain.

In this manuscript we describe how the existent connectivity measures are being applied to EEG recordings for epileptic focus localization and seizure detection.

After a review of the state of the art, we will analyze a benchmark dataset with functional and effective connectivity techniques, introducing some novelties that can be useful to shed light on the spatiotemporal dynamic pattern of seizure origination, spreading and fade-out.

It is worthy to note that recently connectivity in epilepsy is being studied with both functional magnetic resonance imaging (fMRI) alone [3],[4],[5],[6] or coupled to EEG [7].

\section{State of the art}

It has always been clear to the eyes of physicists and mathematicians that the key to understanding epilepsy could be found in the analysis of complex systems and their interactions, and that the various states in which we can observe and record the epileptic brain can leave signatures in the chaotic nature of the data [8],[9],[10],[11] or in their phase space [12].

Given the fact that brain functioning results from the interaction of many complex systems at different scales, it also became clear that insights in the spatiotemporal dynamics of a brain disorder could result from the investigation of how brain regions, near or even distant, are dynamically connected, and that the paths of information transfer throughout the brain can shed light on its functionality and on its breakdown in disease. Indeed to gain better understanding of which neurophysiological processes are linked to which brain mechanisms, structural connectivity in the brain can be complemented by the investigation of statistical dependencies between brain regions (functional connectivity), or of models aimed to elucidate drive-response relationships (effective connectivity) [13]. As opposed to structural connectivity, where the links between brain regions are established by the presence of anatomical fibers, for dynamical (functional and effective) connectivity we consider every site where brain activity is recorded as a node in a graph, connecting the nodes when information is transferred between them.

Even before these definitions and distinctions became so clear (and fashionable), novel methodologies to evaluate directed and symmetric connections were applied to the epileptic brain with two main purposes: localization of the epileptogenic region in order to maximize the probability of success of a surgical intervention, and early and automated seizure detection both for diagnostic purposes and in order to optimize the efficiency of neural stimulation techniques. Approaches for directed connectivity are mostly employed for focus localization, whereas symmetrical measures are more used for seizure detection and prediction. 
From the point of view of information theory, the epileptogenic region is considered to act as a synchronizing source, namely that part of the brain that initiates a transfer of information to other parts of the brain. Considering the recording sites as nodes of a graph, its localization is thus associated to the individuation of those nodes that, in particular around the onset of the seizure, start to behave abnormally as hubs capable to influence the other nodes. The information content is generally confined to specific frequency bands, that's why methods operating in the frequency domain methods are most commonly used.

In order to detect this behavior, directed connectivity is more informative than its undirected counterpart. As we will discuss more in detail later, directed (effective) connectivity is inferred by looking at how the performance of a predictive model changes when information about the different components of a dynamical system is added or removed from it. Concerning model-based approaches, the Directed Transfer Function (DTF), introduced in [14] as an extension to the frequency domain of Granger causality [15], was used to infer the source and the direction of propagation of mesial and lateral temporal lobe seizures [16].

This method has been flanked by other algorithms in view of improving its performance: in [17] the interpretation of DTF results in order to localize the epileptic focus was improved by single class support vector machine, whereas in [18] the optimal frequency to be investigated by DTF was obtained using wavelets. In order to track the evolution of connectivity over time, adaptive methods have been developed. An extension of DTF, ADTF, and an investigation of different variations of it, is applied in [19]. Another time-varying adaptive method, short-time direct DTF [20], is used in [21]. This last study proposes a very promising approach, that consists of evaluating connectivity between partially-dependent component subspaces of an infomax independent component analysis (ICA, [22]) model trained on data from different brain states. The cortical regions are selected using a Bayesian algorithm, and then projected back to the cortical surface for visualization. The reason to do this is to eliminate volume-conduction effects and to reduce dimensionality. It would be especially interesting to apply this approach also to scalp data.

Another measure to detect directed connections in the frequency domain, Partial Directed Coherence (PDC), was introduced in [23]. It has been used to identify epileptogenic regions in [24] and [25]. In section we will present the two methods and the differences between them.

Apart from the studies that focus on frequency domain, some studies have explored connectivity in the time domain. A method based on the analysis of the residual covariance matrix of a multichannel autoregressive model was proposed in [26]. In [27] Granger causality has been used in an animal model to study information transfer between distant regions of interest, in order to assess abnormal brain activity during a spontaneous seizure onset.

A modification of Granger causality, involving canonical correlation analysis, was applied to both scalp and intracranial recordings, filtered in a specific band of interest, in [28]. In this case an asymmetry in the connectivity structure was reported, which could reveal the existence of an epileptic focus even in the absence of ongoing seizure activity.

In all the previous studies based on an autoregressive model, the model order has to be chosen according to some criteria. The most popular are Akaike Information Criterion [29] and Bayesian Information Criterion [30]. Other possible choices are the Hannan-Quinn Criterion [31], or a strategy based on machine learning, namely cross-validation [32].

Together with focus localization, the connectivity approach has been used to validate specific hypotheses on the existence of networks that underlie seizures, following the original idea proposed in [33]. In [34] specific graph signatures were associated with different brain states, including epilepsy. In [35] the connectivity matrices obtained by coherence underwent graph theoretical analysis to detect the network architecture associated with seizures.

In [36] the authors hypothesized that the region assumed to generate seizures was a network with variable excitability. Then they considered a simple computational model on two populations in order to quantify functional and effective connectivity measures on them. They first stated that rapid discharges and hyper-excitability between the two populations could be obtained by different model structures such as unidirectional or bidirectional coupling. They also agreed that only nodes with high levels of excitability were worthwhile to be considered as elements of the fast onset activity. So, when one of the two populations presented hyper-excitability then it was believed to be able to generate a fast activity itself. With an example the authors drew the attention to how connectivity (effective in this case) can be interpreted and how the notion of rapid discharges and propagation should be clarified. So an epileptic network can include nodes that are able to generate a rapid discharge and other ones that are driven from the former one to an altered excitability and to the capability of generating discharges themselves.

In [37] the generation of an epileptic seizure out of a network structure was investigated. It was hypothesized that whenever an EEG discharge was present, it was driven by a pattern of brain networks. To support this, a brain network of four regions of interest with some established connections of the same strength were generated. The 
authors then investigated the differences of varying these connections between the regions of the network versus an introduction of a new brain region in the network, which is characterized by an abnormal activity. In the case of introduction of a region with an abnormal activity, and depending on the connections that they set between each of the regions, there was a rise of focal, primary or secondary generalized seizures. When the connectivity was weakened, an increase in the frequency typical of seizure activity was observed.

\section{Seizure prediction and detection}

A connectivity based approach has also proven useful in improving the early detection or prediction of seizures, with respect to considering the complexity of a signal at a certain time. The general motivation behind the first studies in this sense was that the information gathered studying the complexity of an electroencephalographic time series could be augmented by considering how this complexity is modulated by the interactions with other time series.

In [38] nonlinear time series analysis was used for early prediction of an impending seizure. The basic idea was the timely identification of transitions of the system from lower to higher complexity and from asynchronous to synchronous activity on longer time scales. The EEG recordings from the epileptogenic region of the brain indicated significant changes in nonlinear dynamics up to several minutes prior to the clinical seizure onset as compared to other recording sites.

Sometimes the volume conduction effects could lead to misleading results in several connectivity measures, in particular those relying mostly on the amplitude of the signal; for this reason phase coherence, a method quantifying the symmetrical dependencies between oscillating signals, was successfully applied in [39]. In this case this bivariate measure was reported to be more efficient compared to univariate measures in predicting an upcoming seizure. This result is also described and expanded in [40], and thoroughly validated in [41]. In this last study a validation of 30 univariate and bivariate prediction algorithms found in the literature was conducted, starting from the idea that many prediction algorithms lacked in statistical validation as they did not test the specificity of seizure precursors. Bivariate measures showed high statistical performance with a constant baseline, highlighting pre-ictal states even $240 \mathrm{sec}$ before the seizure onset. Univariate methods were statistically significant on a seizure wise basis, with an adaptive baseline, identifying pre-ictal changes from 5 to $30 \mathrm{sec}$ before the seizure. The authors concluded that a combination of univariate and bivariate methods comprising both linear and non-linear approaches provides a promising solution for seizure prediction.

Phase coherence, joint with another synchronization measure, lag synchronization, was also discussed in [42], where the issue of the variability between patients was raised. Phase synchronization methods remain among the most successfully applied [43].

A wavelet-based and frequency specific non-linear similarity index (WNSI) has been applied in [44] on intracranial recordings to predict epileptic seizures. The fact that the EEG data pattern is not modified by the application of a wavelet transform is considered an advantage of this measure. This characteristic allows to investigate the nonlinear dynamics of EEG patterns.

In the same direction as [41], in [45] the predictive power of prediction algorithms was tested against well established null hypotheses. They concluded that the time surrogates approach outperforms analytic performance estimates under controlled conditions. This is due to the initial construction of seizure prediction surrogates which is not restricted by specificity, sensitivity or performance definitions while analytic performance estimates are constructed as functions of false positive rates.

In [46] we find another example of exploiting network structure to improve the research on early seizure detection. This method combines spectral techniques with matrix theory. From multi-site stereo-eeg (SEEG) recordings in epileptic patients, time windows of the same length were considered and connectivity matrices were built for every second window, in order to describe the time dependent correlation between channels. For each one of those matrices, the Singular Value Decomposition is computed in order to track the dominant structure of each matrix over time. The main target was to detect changes of those matrices in pre-ictal and ictal cases. The first singular vector, which represents the dominant effect of each matrix, was sought in both pre-ictal and ictal cases. Then the inner product of the calculated mean ictal singular vector and the first singular vector were calculated for each time window. The results showed significant differences with higher inner products of the singular vectors throughout the seizure time and the average ictal vector compared to one calculated throughout the pre-ictal period.

This idea was exploited and optimized in [47], in which the time course of the maximum singular value of the connectivity matrix obtained by spectral coherence underwent a fast detection procedure which minimized the false 
positives. This approach introduced one of the key ideas applied in the present study.

It is worthy to note that the measure described in [39], and applied with more detail in [41] described a decrease of the connection strength during the seizure, while for example in [48] and [25] epilepsy is described as a more organized state with increased coupling strength. This could indeed be related to the difference between coupling measures based on phase and amplitude. A critical discussion of amplitude versus phase coupling in epilepsy is contained in [49].

\section{Information theory}

An issue that we find particularly relevant is that all these measures could be interpreted in terms of information transfer, allowing an improved mathematical tractability and a generalized framework. This choice is further justified by the fact that Granger causality and its equivalents in the frequency domain do not measure coupling strength but predictive information transfer.

Palus et al. [50] interpreted synchronization as an adjustment in information rate, associating different amounts of exchanged information to the ictal and interictal phase.

The discussion about formulation of DTF in terms of information transfer has been started by Eichler [51], and extended and generalized to PDC in [52]. Barnett et al. [53] have shown that under the assumption of Gaussian distribution of the variables Granger causality is equivalent to Transfer Entropy (TE), a model-free measure of directed connectivity [54]. This result has been used to optimize Granger causality analysis to infer connectivity in high dimensional datasets, as those encountered in epilepsy analysis, in [55]. Connectivity patterns in the epileptic brain obtained by TE are reported in [56],[57] and [58]. It is important at this point to note that there is ample evidence that neural data are not Gaussian distributed (see for example the discussion of this topic in [59]). Even if for neural data the equivalence does not exactly hold (preventing for example to measure GC or PDC in bits), we believe that this unified framework can be beneficial both for the computational/methodological part, and for the interpretation of the results, keeping in mind that model free methods such as the entropy based ones ensure indeed more general validity.

\section{An illustrative example}

In this section we apply coherence, DTF and PDC to a benchmark dataset, starting from the approach employed in [47] for seizure detection, but also trying to incorporate information on the focus localization, tracking the maximum singular value also on individual rows and columns of the directed connectivity matrices.

We recapitulate the main methods and then present some results.

\section{Methods}

Coherence

Coherence is a measure indicating the degree of linear association between two time series in the frequency domain. Given two time series $\mathrm{X}$ and $\mathrm{Y}$, coherence is given by:

$$
C_{f}=\frac{(\text { cross power spectrum }(X, Y))^{2}}{\text { power spectrum }(X) \text { power spectrum }(Y)}=\frac{\left|S_{x y}(f)\right|^{2}}{S_{x x}(f) S_{y y}(f)} .
$$

Coherence has been extensively used to detect and quantify the interaction of two time series in the frequency band. However, coherence does not allow inferring directionality of the information transfer and is largely influenced by amplitude effects.

\section{Granger causality}

The introduction of directed connectivity measures such as Granger Causality (GC) [15] in the time domain and its analogues in the frequency domain, Directed Transfer Function (DTF) [14] and Partial Directed Coherence (PDC) [23] represented a great improvement in defining the direction of the influences among time series, and are 
increasingly being applied to neuroscience.

GC was initially introduced in the field of econometrics. Its key idea lies in the improvement of the performance of a predictive model of a time series given some of its past values when information from the past of another time series is incorporated in it. The original model was a bivariate autoregressive model given by:

$$
\begin{aligned}
& X=\sum_{j=1}^{p} A_{11}(j) X(t-j)+\sum_{j=1}^{p} A_{12}(j) Y(t-j)+e_{1}(t) \\
& Y=\sum_{j=1}^{p} A_{21}(j) X(t-j)+\sum_{j=1}^{p} A_{22}(j) Y(t-j)+e_{2}(t)
\end{aligned}
$$

with $A_{i k}$ being the model parameters and $e_{i}$ the white noise where $i, k=1,2$.

Granger causality quickly became a standard tool for inferring directed relationships between time series. However, in its original formulation as a bivariate measure it can lead to erroneous results and false positives especially when the channels are fed from common signal sources. The first approach in the literature for applying Granger causality in a multivariate case was, proposed by Geweke [60].

Moreover, the increasing need in analysis of biomedical series, which display evident signatures in rhythms at a given frequency, together with the fact that the use of GC on filtered signals is questionable [61],[62] renders the use of equivalent measures in the frequency domain indispensable.

\section{Directed transfer function}

The Directed transfer function was formulated in the framework of an autoregressive model (AR) in the frequency domain. It is developed as a measure able to study the interrelation between two signals in relation to all other signals. The AR model is characterized by:

$$
\sum_{j=0}^{p} \hat{A}_{j} x_{t-j}=e_{t}
$$

where $x_{t}=\left(x_{1, t}, x_{2, t}, \ldots x_{k, t}\right)$ is a vector of a $k$ channel process, $e_{t}=\left(e_{1, t}, e_{2, t}, \ldots, e_{k, t}\right)$ is a vector of multivariate uncorrelated white noise process, and $\hat{A_{1}}, \hat{A_{2}}, \ldots, \hat{A_{p}}$ are the $k \mathrm{x} k$ matrices of model coefficients.

Multiplying both sides of (4) by $x_{t-s}^{T}$ and taking expectation values, gives the coefficients $\hat{A}_{i}$. This leads to the following equation:

$$
\hat{R}(-s)+\hat{A}_{1} \hat{R}(1-s)+\ldots+\hat{A}_{p} \hat{R}(p-s)=0
$$

where $\hat{R}(s)=E\left[x_{t}, x_{t+s}^{T}\right]$ is the covariance matrix for a lag $s$.

In order to investigate the spectral properties between the signals, Fourier transformation is applied to equation (4) where the transform functions are of the form:

$$
\hat{X}(z)=\hat{H}(z) \hat{E}(z)
$$

with

$$
\hat{H}(z)=\left(\sum_{j=0}^{p} \hat{A}_{j} e^{-i 2 \pi f \Delta t}\right)^{-1}
$$

DTF is usually normalized with respect to incoming influence so after normalization it takes the form:

$$
\gamma_{i j}^{2}=\frac{\left|H_{i j}(f)\right|^{2}}{\sum_{m=1}^{k}\left|H_{i m}(f)\right|^{2}}
$$


Consequently, the element $H_{i j}(f)$ of the matrix $H(f)$ describes the connection between the $j$-th input and the $i$-th output of the system (transmission from channel $j \rightarrow i$ ). When normalization is applied DTF takes values in the interval $[0,1]$ where a high value indicates a consistent information transfer in the direction $j \rightarrow i$ and a low value indicates little or no transfer. In the literature different strategies for normalization of DTF (or no normalization at all) are proposed depending on whether the main interest is in the direction rather than in the ratio of influences $[63],[64],[51]$.

Even though DTF was initially introduced in [14] as a bivariate measure, there are studies applying it to multivariate systems. In the latter cases the use of DTF can reveal cascade transfers e.g for channels a, b, c if $a \rightarrow b \rightarrow c$ and in this case DTF also detects propagation from $a \rightarrow c$. [63] and [65] propose a modified version of DTF, the directed DTF (dDTF) which was able to detect whether a connection between two nodes is mediated by a third one. The dDTF is a combination of the partial coherence function and of the original definition of DTF, emphasizing only direct connections.

\section{Partial directed coherence}

When we have $K$ simultaneously recorded signals, the information transfer can also be computed directly by the Fourier transform of model coefficients of (4). This leads to the Partial directed coherence (PDC) which is defined within the framework of Granger causality in the frequency domain and is a measure of the interaction of two time series when the effect of the remaining $K-2$ time series is removed. It is designed to describe the relationship of multivariate time series based on the decomposition of multivariate coherences computed from multivariate AR models.

PDC from channel $j$ to channel $i$ is given by:

$$
\pi_{i j}(f)=\frac{\hat{A}_{i j}(f)}{\sqrt{\hat{a}_{j}^{H}(f) \hat{a}_{j}(f)}}
$$

where the superscript $H$ stands for Hermitian transpose and $\hat{A}_{i j}$ is calculated as:

$$
\hat{A}_{i j}(f)=\left\{\begin{array}{c}
1-\sum_{r=1}^{p} \hat{a}_{i j}(r) e^{-i 2 \pi f r}, i=j \\
-\sum_{r=1}^{p} \hat{a}_{i j}(r) e^{-i 2 \pi f r}, i \neq j
\end{array}\right.
$$

The PDC is normalized with respect to the outgoing influences resulting in:

$$
\begin{gathered}
0 \leq\left|\pi_{i j}(f)\right|^{2} \leq 1 \\
\sum_{i=1}^{N}\left|\pi_{i j}(f)\right|^{2}=1
\end{gathered}
$$

The PDC is able to rank the strength of the direct interactions of a channel $\mathrm{j}$ to the other channels which are receiving information from $\mathrm{j}$, a fact that renders it a useful tool for the detection of putative information sinks [66].

Reporting what is clearly explained in [65], an important difference between DTF and PDC lies in the normalization: DTF is normalized with respect to the structure that receives the signal, while PDC is normalized with respect to the structure that sends out the signal. Summarizing, we can state that DTF measures influence as the amount of information being transferred between two time series through all (direct and indirect) transfer pathways, relative to the total influence on the target; the PDC measures directed predictive information transfer from the source to the target through the direct transfer pathway only, relative to the total information leaving the source. We note that this dual interpretation highlights advantages and disadvantages of both measures. DTF has a meaningful physical interpretation as it measures predictive information transfer as the amount of signal power transferred from one process to another, but cannot distinguish between direct and indirect influences measured in the frequency domain. Conversely, PDC clearly reflects the underlying interaction structure as it provides a one-to-one representation of direct causality, but is hardly useful as a quantitative measure because its magnitude quantifies the information flow through the inverse spectral matrix elements (which are not easily interpreted in terms of power spectral density).

\section{Connectivity matrix and Singular value decomposition}

A connectivity matrix was built from each data segment and for all different measures. From these connectivity matrices the incoming, outgoing and total information from each node was then extracted. Of course the distinction 
between incoming and outgoing information is applicable only to directed measures, thus not to coherence.

The computation of inflow and outflow of information from each channel can provide information on which channels can be potential sinks (receiving information from other channels) or sources (sending out information to the other channels) of information.

The rank of the connectivity matrix indicates the number of the linearly independent rows or columns. So, in cases that the connections between the channels are strengthened the rank of the matrix drops. In contrast, when the connections are weak the rank increases. Thus, tracking the rank of the connectivity matrices helps to detect the transition to a more organized state in brain activity and thus, gathering relevant information on the dynamics of the seizure onset.

Singular Value Decomposition (SVD) is used to define an $m \times n$ matrix $A$ as follows: $A=U S V^{*}$, where $U$ is a $m \times \mathrm{x} m$ unitary matrix whose columns are the eigenvectors of the matrix $A A^{*}, S$ is a $m$ x $n$ matrix with non-zero $r$ diagonal entries, with $r$ representing the rank of $A$ and $V$ a $n \times n$ unitary matrix whose columns represent the eigenvectors of the matrix $A^{*} A$. $\left({ }^{*}\right)$ in all cases stands for the conjugate transpose.

We can characterize the connectivity structure by looking at the maximum of the singular values contained in the matrix $S$ (MSV) as described in [47]. Here, as in [47] we apply this analysis to the coherence, but we extend it also to directed measures (DTF and PDC) with the aim of efficiently mapping functional and effective connectivity both in space and time.

\section{Data}

We consider a dataset consisting of scalp and intracranial EEG recordings from a patient with refractory epilepsy containing 5 seizures from Ghent University Hospital. The intracranial electroencephalographic seizures onsets were marked by the epileptologists (KV and $\mathrm{PB}$ ). The dataset contained 27 scalp electrodes, 48 cortical subdural electrodes, divided into a $4 \times 8$ array (TG $1-32$ ) and a $2 \times 8$ array (SG $1-16$ ), and a depth electrode with 12 contacts (RD 1-12). Based on the invasive video EEG monitoring the epileptogenic zone was localized within a dyplastic insular lesion on the right side. Following resective surgery the patients is now seizure free for more than 6 months. A scheme with the position of the intracranial electrode is shown in figure 1 . The sampling frequency of the recorded EEG signals is $256 \mathrm{~Hz}$. We extracted from the EEG series a segment that starts $120 \mathrm{~s}$ before the electroencephalographic seizure onset (pre-ictal) and ends $120 \mathrm{~s}$ after the end of the seizure (post-ictal).

Since epileptiform focus activity is concentrated in frequency bands which are patient-specific, we first identified this band in order to concentrate our analysis on it. We did this by applying a general linear model to ictal and interictal data filtered in the different bands to find out where the maximal differences were. For the analyzed dataset the chosen band was the Beta-Gamma band $([1245] \mathrm{Hz})$. In order to track the modulation of the connectivity in time we computed the connectivity matrix in time windows of 5 seconds sliding with a step of 1.5 seconds. The connectivity matrices were computed using spectral coherence as well as two directed measures (DTF and PDC, optimized for evaluating outgoing and incoming information respectively). For each matrix we evaluated the maximum singular value. As an innovation with respect to [47], we obtained this measure not only for the global matrix, but also for the single rows and columns, representing for each channel the outgoing and incoming information respectively. This allows to gather additional information on the spatiotemporal pattern of the seizure.

\section{Results}

We tracked the maximum singular value described above by observing its evolution over time. In order to evaluate the performance of each one of the measures previously introduced, we computed both the total flow for all the nodes and the inflow and outflow for each one of them.

For the 27 scalp electrodes, coherence captured a drop in the maximum singular value before the time marked as intracranial electroencephalographic onset, followed by a sharp increase. The MSV remained high also after the end of the seizure (figure 2 top). High values of the maximum singular value indicate less diversity but stronger components which is in agreement with the concept that during the seizure the brain enters a more organized state. The interpretation of the momentary increase in independence of the nodes resulting in the initial drop in MSV, which could be possibly used for early seizure detection, will require further validation and discussion. For the 60 cortical contacts there is a similar trend compared to the one in the scalp electrodes, with an increased maximum singular value during the electroencephalographic onset. However coherence in case of cortical electrodes proved a bit slower to detect the seizure onset compared to the scalp electrodes, and the MSV returned earlier to baseline 
RD01 TG01 TG02 TG03 TG04 TG05 TG06 TG07 TG08

$\mathrm{RD} 02$

$\mathrm{RD} 03$

$\mathrm{RD} 04$

$\mathrm{RD} 05$

$\mathrm{RD} 06$

$\mathrm{RD} 07$

$\mathrm{RD} 08$

RD09

RD10

TG09 TG10 TG11 TG12 TG13 TG14 TG15 TG16

TG17 TG18 TG19 TG20 TG21 TG22 TG23 TG24

TG25 TG26 TG27 TG28 TG29 TG30 TG31 TG32

RD11

RD12

\section{SG01 SG02 SG03 SG04 SG05 SG06 SG07 SG08 \\ SG09 SG10 SG11 SG12 SG13 SG14 SG15 SG16}

FIG. 1: Scheme with the location of the intracranial electrodes. On the left the depth electrode $(R D 1-12)$ in the right insular region. On the right upper part a 32-contact right temporal grid (TG1-32), below a 16-contact right frontoparietal grid $(S G 1-16)$.

values (see figure 2 bottom for an example).

For the outgoing information in the scalp electrodes we observed a decrease in the maximum singular value around the onset and immediate increase after the end of it. The drop in the MSV indicates that the nodes are more independent during the onset while they become more correlated immediately after the end of the seizure.

We observed more variation in the source activity measured by DTF for each cortical node. Indicatively, for some contacts there is a clear drop of the MSV at the electroencephalographic onset (figure 3, top left), for others a clear drop after the seizure (figure 3, top right) while for others an increase of the MSV after the end of the seizure (figure 3 , bottom).

As an illustrative example, in figure 4 we report the scalp map of the percentage variation of MSV for DTF during seizures with respect to baseline.

The normalized partial directed coherence is described by the ratio of the outgoing information from a node $\mathrm{j}$ to a node $\mathrm{i}$ and the total outgoing information from node $\mathrm{j}$. For both scalp and cortical electrodes, PDC calculated sink activity within the interval of the electroencephalographic borders set by the epileptologists. The total incoming information quantified by PDC, shows variability among the 60 cortical contacts. The general trend in the 12 depth contacts is in agreement with results of the total flow, as an increase in the MSV is observed for each one of them (figure 5, top left). A decrease during the seizure and a raise after it is detected for some of the subdural contacts (figure 5, top right) while a clear peak and then a drop after the end of the electrographic seizure is indicated in others (figure 5 bottom).

In figure 6 we report the map of the percentage variation of MSV for PDC at the onset of the seizure and 10 seconds after with respect to baseline across the intracranial contacts. The maximum percentage variation is reported at one extremity of the depth electrode (RD), confirming the presence of the seizure onset in the deep structures. After 10 seconds we observed an increase also in the cortical electrodes, indicating spreading seizure activity. A similar pattern is observed for the outgoing connections as measured by PDC (figure 7), but in this case the pattern is more stable 

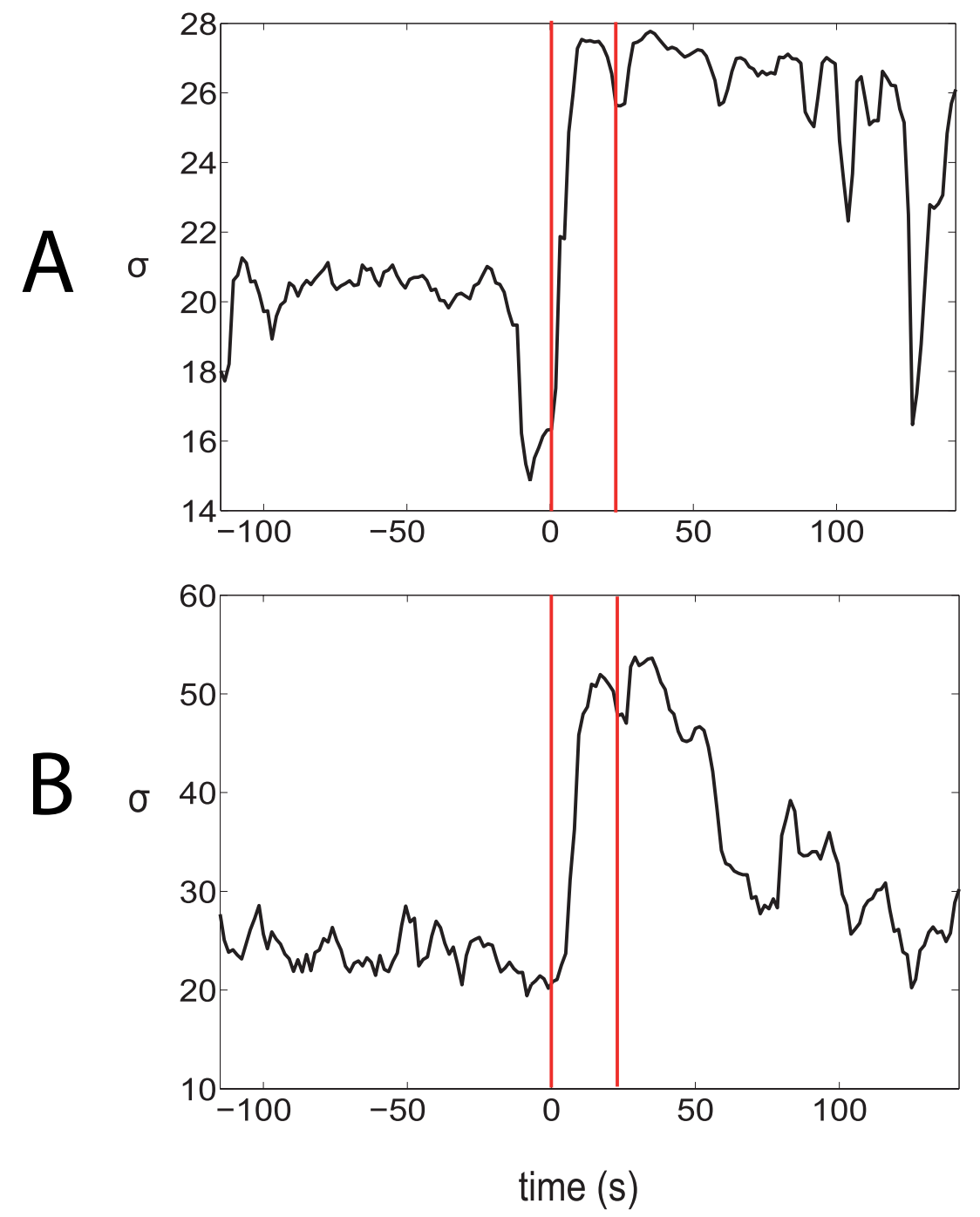

FIG. 2: Evolution of the maximum singular value $\sigma$ over time(s). (A): Coherence measured over the 27 scalp electrodes. An increase of $\sigma$ is captured around the intracranial electroencephalographic onset indicating less diversity and more dominant components. (B): Coherence measured over the 60 cortical electrodes. A similar pattern with an earlier increase is observed in the scalp electrodes.

during the seizure. We can interpret this difference in view of a recent result [67] showing that in a hierarchical network the information going out from each node increases with the number of neighbors while the incoming information stays more or less constant.

Moreover, and surprisingly, scalp electrodes are those for which the variation in the connectivity occurs the earliest. Previous studies [68] have reported that the predominance of global synchronization and overall volume conduction induce a great variability of these scalp patterns, but this early modification of the dynamical connectivity could open interesting perspectives for the development of therapeutic measures that may not require invasive recordings and give hints also on the location in space and time of the seizure termination.

\section{Conclusions}

We have provided an overview of the methods that explore dynamical connectivity in human EEG recordings to understand the physiological mechanisms underlying epilepsy, and also their application in the detection of the epileptogenic region and prediction of seizure activity. We have shown that, for the analyzed case, some measures that have been previously employed for seizure detection can be also useful for focus localization. Furthermore the 

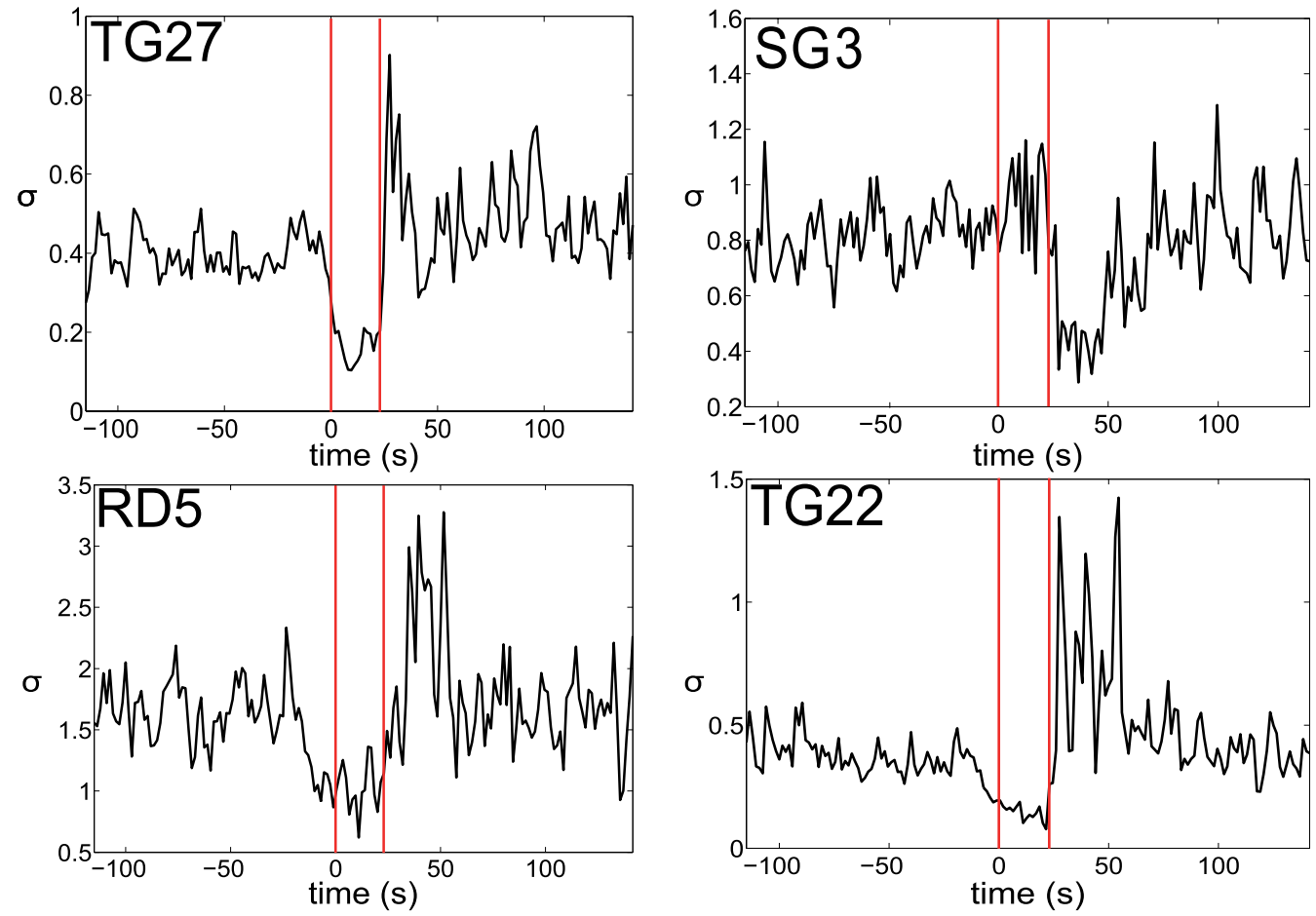

FIG. 3: Examples of outgoing information captured by DTF in some cortical contacts for a single seizure (red lines indicate electroencephalographic onset and termination). Some contacts present a clear drop of $\sigma$ at the electroencephalographic onset, indicating that the components become more random during the seizure (top right), where others present this drop straight after the electroencephalographic onset (top left). In other cases DTF captures a significant rise of $\sigma$ straight after the end of the electroencephalographic onset (bottom)
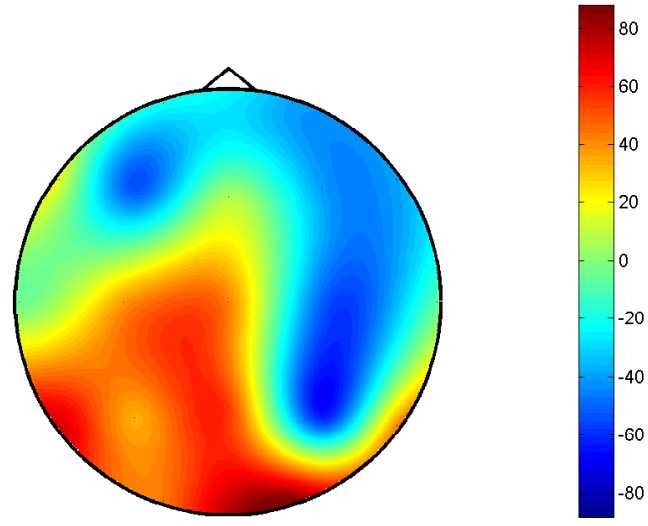

FIG. 4: Percentage variation of the MSV for DTF in scalp electrodes, averaged over 5 seizures.

employed algorithms are fast enough to allow for real-time application, thus making them amenable to clinical use. This paper presents preliminary results and its purposes do not reach as far as evaluating their diagnostic value. The point we wish to make is that an integrated spatiotemporal approach, as well as a unified framework such as information theory, may represent an optimal strategy for the future of the analysis of epilepsy from a dynamical network perspective. 

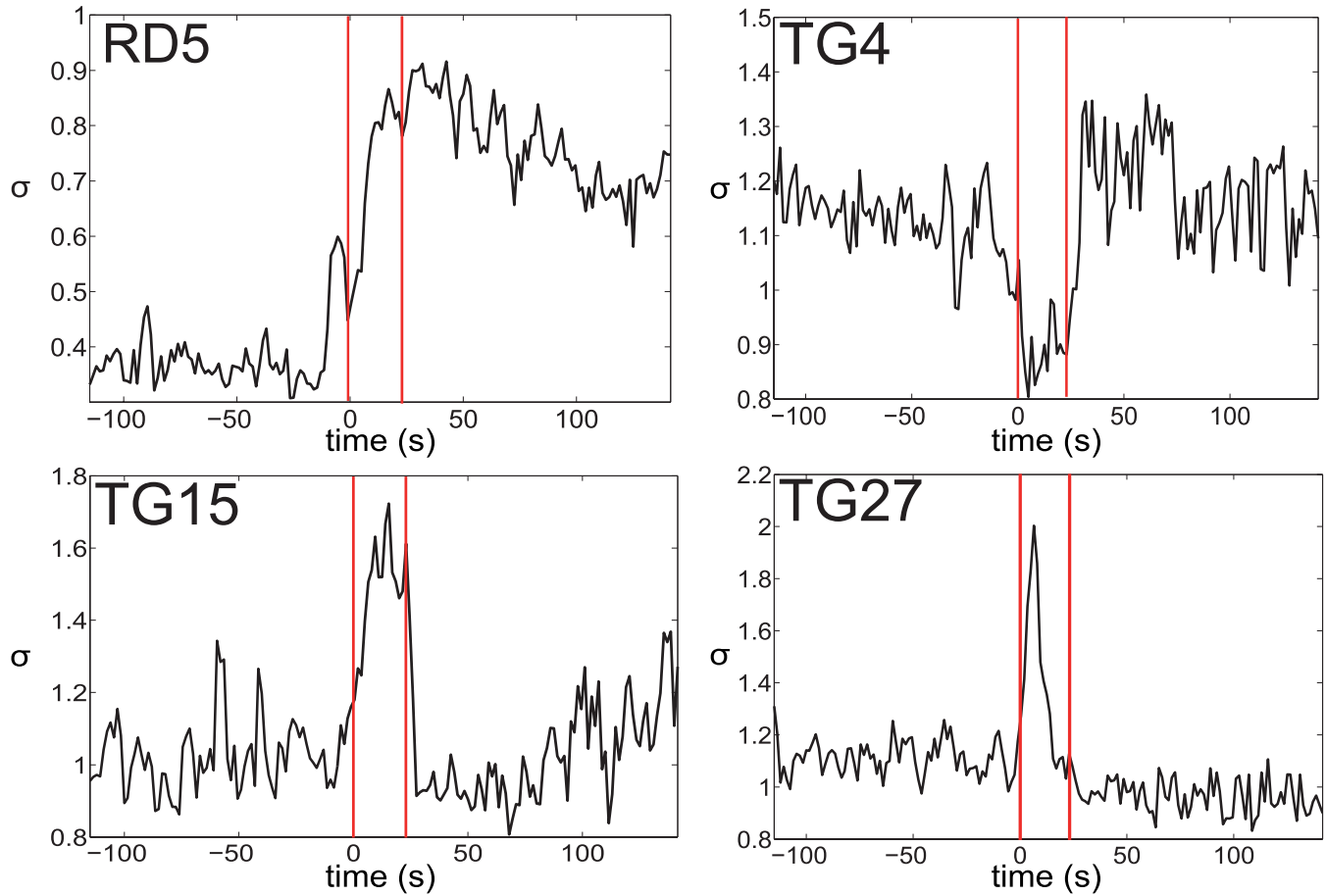

FIG. 5: Examples of outgoing information captured by PDC in some cortical contacts for a single seizure (red lines indicate electroencephalographic onset and termination). Some contacts display an increase of $\sigma$ at the electroencephalographic onset indicating more dominant components (top left), where others present lower values during the seizure and a raise immediately after it (top right). For some, high incoming activity is captured at the electroencephalographic onset (bottom)
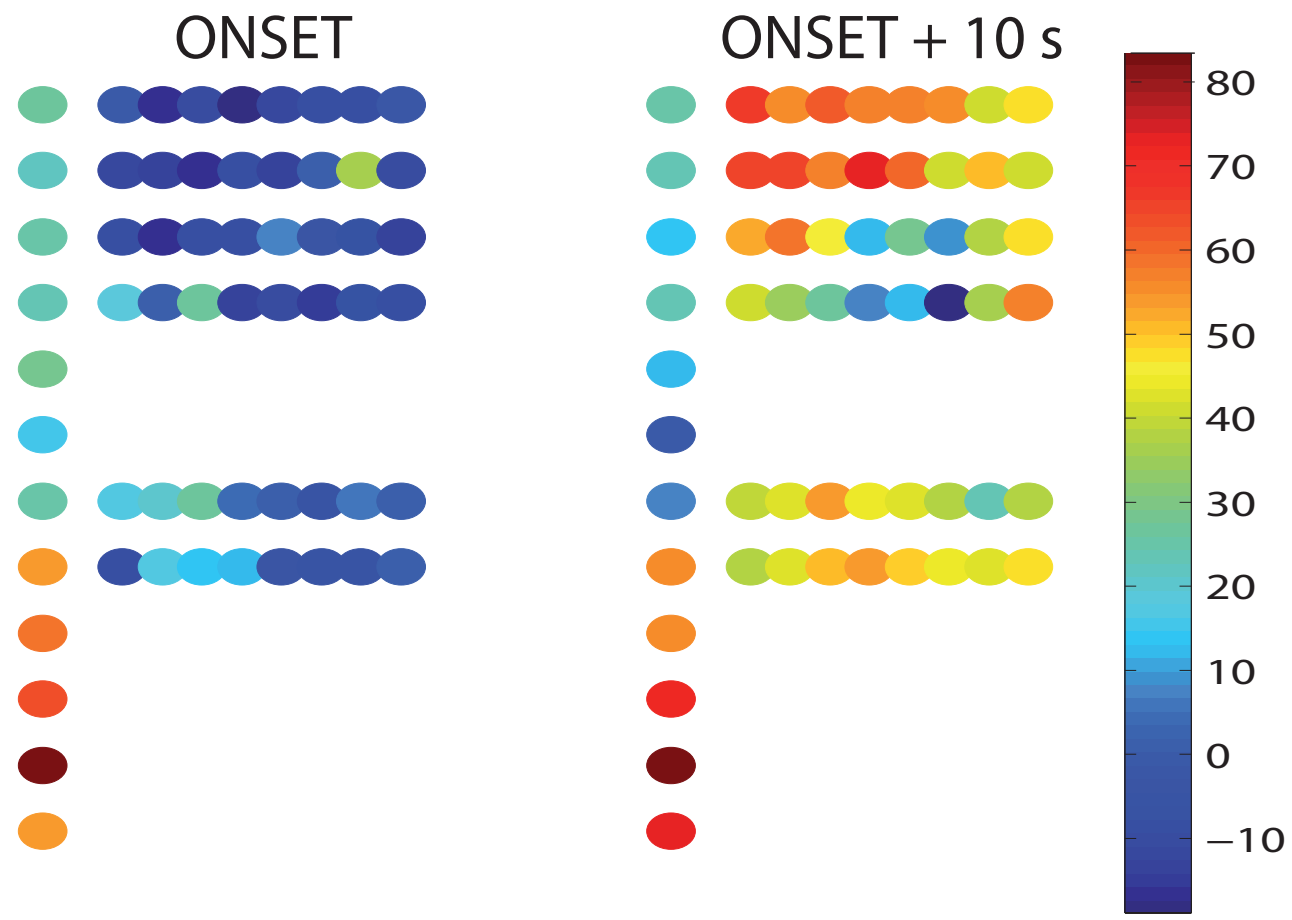

FIG. 6: Percentage variation of the MSV for PDC in intracranial electrodes, at the onset of the seizure and 10 seconds after the onset, averaged over 5 seizures. The position of the electrodes reflects the scheme reported in figure 1 

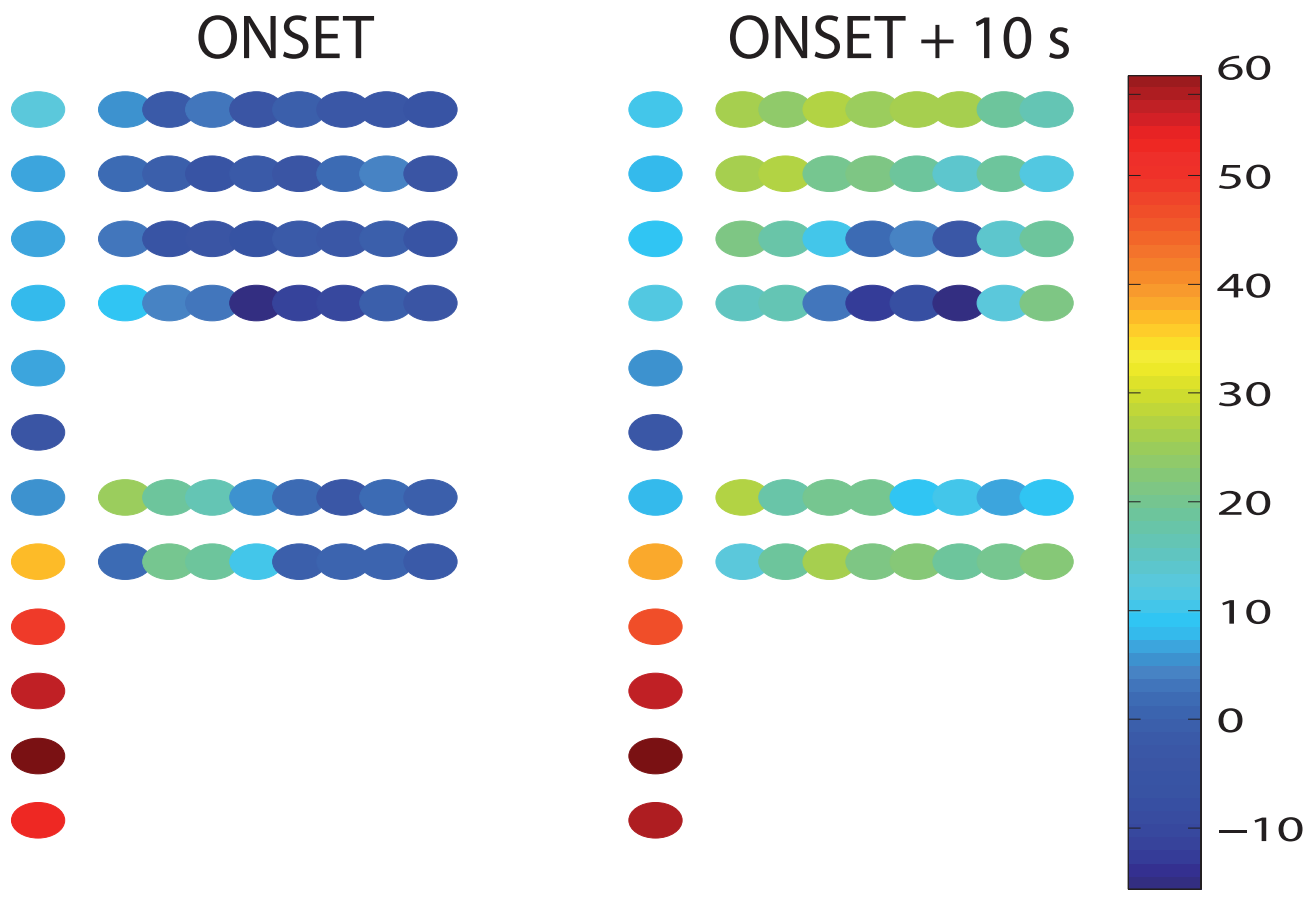

FIG. 7: Percentage variation of the MSV for PDC in intracranial electrodes, at the onset of the seizure and 10 seconds after the onset, averaged over 5 seizures. The position of the electrodes reflects the scheme reported in figure 1 
[1] F. Mormann, R. G. Andrzejak, C. E. Elger, and K. Lehnertz, "Seizure prediction: the long and winding road.," Brain : a journal of neurology, vol. 130, pp. 314-33, Feb. 2007.

[2] B. C. Jobst, T. M. Darcey, V. M. Thadani, and D. W. Roberts, "Brain stimulation for the treatment of epilepsy.," Epilepsia, vol. 51 Suppl 3, pp. 88-92, July 2010.

[3] X. Zhang, F. Tokoglu, M. Negishi, J. Arora, S. Winstanley, D. D. Spencer, and R. T. Constable, "Social network theory applied to resting-state fMRI connectivity data in the identification of epilepsy networks with iterative feature selection.," Journal of neuroscience methods, vol. 199, pp. 129-39, July 2011.

[4] M. Negishi, R. Martuzzi, E. J. Novotny, D. D. Spencer, and R. T. Constable, "Functional MRI connectivity as a predictor of the surgical outcome of epilepsy.," Epilepsia, vol. 52, pp. 1733-40, Sept. 2011.

[5] F. Pittau, C. Grova, F. Moeller, F. Dubeau, and J. Gotman, "Patterns of altered functional connectivity in mesial temporal lobe epilepsy," Epilepsia, vol. 53, pp. no-no, May 2012.

[6] J. Zhang, W. Cheng, Z. Wang, Z. Zhang, W. Lu, G. Lu, and J. Feng, "Pattern Classification of Large-Scale Functional Brain Networks: Identification of Informative Neuroimaging Markers for Epilepsy," PLoS ONE, vol. 7, p. e36733, May 2012.

[7] T. Murta, A. Leal, M. I. Garrido, and P. Figueiredo, "Dynamic Causal Modelling of epileptic seizure propagation pathways: A combined EEG-fMRI study.," NeuroImage, vol. 62, pp. 1642-1634, May 2012.

[8] L. D. Iasemidis, J. C. Sackellares, H. P. Zaveri, and W. J. Williams, "Phase space topography and the Lyapunov exponent of electrocorticograms in partial seizures.," Brain topography, vol. 2, pp. 187-201, Jan. 1990.

[9] J. P. Pijn, J. Van Neerven, A. Noest, and F. H. Lopes da Silva, "Chaos or noise in EEG signals; dependence on state and brain site.," Electroencephalography and clinical neurophysiology, vol. 79, pp. 371-81, Nov. 1991.

[10] F. H. Lopes da Silva, J. P. Pijn, and W. J. Wadman, "Dynamics of local neuronal networks: control parameters and state bifurcations in epileptogenesis.," Progress in brain research, vol. 102, pp. 359-70, Jan. 1994.

[11] K. Lehnertz and C. E. Elger, "Spatio-temporal dynamics of the primary epileptogenic area in temporal lobe epilepsy characterized by neuronal complexity loss.," Electroencephalography and clinical neurophysiology, vol. 95, pp. 108-17, Aug. 1995 .

[12] J. Martinerie, C. Adam, M. Le Van Quyen, M. Baulac, S. Clemenceau, B. Renault, and F. J. Varela, "Epileptic seizures can be anticipated by non-linear analysis.," Nature medicine, vol. 4, pp. 1173-6, Oct. 1998.

[13] K. J. Friston, "Functional and Effective Connectivity: A Review," Brain Connectivity, vol. 1, pp. 13-36, Jan. 2011.

[14] M. J. Kaminski and K. J. Blinowska, "A new method of the description of the information flow in the brain structures," Biological Cybernetics, vol. 65, pp. 203-210, July 1991.

[15] C. W. J. Granger, "Investigating Causal Relations by Econometric Models and Cross-Spectral Methods," Econometrica, vol. 37 , no. 3, pp. $424-38,1969$.

[16] P. J. Franaszczuk and G. K. Bergey, "Application of the directed transfer function method to mesial and lateral onset temporal lobe seizures.," Brain topography, vol. 11, pp. 13-21, Jan. 1998.

[17] B. Swiderski, S. Osowski, A. Cichocki, and A. Rysz, "Single-class SVM and directed transfer function approach to the localization of the region containing epileptic focus," Neurocomputing, vol. 72, pp. 1575-1583, Mar. 2009.

[18] C. Wilke, W. van Drongelen, M. Kohrman, and B. He, "Neocortical seizure foci localization by means of a directed transfer function method.," Epilepsia, vol. 51, pp. 564-72, Apr. 2010.

[19] P. van Mierlo, E. Carrette, H. Hallez, K. Vonck, D. Van Roost, P. Boon, and S. Staelens, "Accurate epileptogenic focus localization through time-variant functional connectivity analysis of intracranial electroencephalographic signals.," NeuroImage, vol. 56, pp. 1122-1133, June 2011.

[20] A. Korzeniewska, C. M. Crainiceanu, R. Kuś, P. J. Franaszczuk, and N. E. Crone, "Dynamics of event-related causality in brain electrical activity.," Human brain mapping, vol. 29, pp. 1170-92, Oct. 2008.

[21] T. Mullen, Z. A. Acar, G. Worrell, and S. Makeig, "Modeling cortical source dynamics and interactions during seizure.," Proceedings of the Annual International Conference of the IEEE Engineering in Medicine and Biology Society, vol. 2011, pp. 1411-4, Jan. 2011.

[22] A. J. Bell and T. J. Sejnowski, "An information-maximization approach to blind separation and blind deconvolution," Neural Computation, vol. 7, pp. 1129-1159, 1995.

[23] L. A. Baccalá and K. Sameshima, "Partial directed coherence: a new concept in neural structure determination.," Biological cybernetics, vol. 84, pp. 463-474, May 2001.

[24] D. Y. Takahashi, L. A. Baccal, and K. Sameshima, "Connectivity Inference between Neural Structures via Partial Directed Coherence," Journal of Applied Statistics, vol. 34, no. 10, pp. 1259-1273, 2007.

[25] G. Varotto, E. Visani, L. Canafoglia, S. Franceschetti, G. Avanzini, and F. Panzica, "Enhanced frontocentral EEG connectivity in photosensitive generalized epilepsies: a partial directed coherence study.," Epilepsia, vol. 53, pp. 359-67, Feb. 2012.

[26] P. J. Franaszczuk and G. K. Bergey, "An autoregressive method for the measurement of synchronization of interictal and ictal EEG signals.," Biological cybernetics, vol. 81, pp. 3-9, July 1999.

[27] A. J. Cadotte, T. B. DeMarse, T. H. Mareci, M. B. Parekh, S. S. Talathi, D.-U. Hwang, W. L. Ditto, M. Ding, and P. R. Carney, "Granger causality relationships between local field potentials in an animal model of temporal lobe epilepsy.," Journal of neuroscience methods, vol. 189, pp. 121-9, May 2010.

[28] G. R. Wu, F. Chen, D. Kang, X. Zhang, D. Marinazzo, and H. Chen, "Multiscale causal connectivity analysis by canonical 
correlation: theory and application to epileptic brain.," IEEE transactions on bio-medical engineering, vol. 58, pp. 3088-96, Nov. 2011.

[29] H. Akaike, "A new look at the statistical model identification," IEEE Transactions on Automatic Control, vol. 19, pp. 716723, Dec. 1974.

[30] G. Schwarz, "Estimating the Dimension of a Model," The Annals of Statistics, vol. 6, pp. 461-464, Mar. 1978.

[31] B. G. Quinn, "Order determination for a multivariate autoregression," Journal of the Royal Statistical Society. Series B (Methodological), vol. 42, no. 2, pp. pp. 182-185, 1980.

[32] R. Kohavi, "A Study of Cross-Validation and Bootstrap for Accuracy Estimation and Model Selection,"

[33] S. Spencer, "Neural Networks in Human Epilepsy: Evidence of and Implications for Treatment," Epilepsia, vol. 43, pp. 219-227, Mar. 2002.

[34] S. C. Ponten, F. Bartolomei, and C. J. Stam, "Small-world networks and epilepsy: graph theoretical analysis of intracerebrally recorded mesial temporal lobe seizures.," Clinical neurophysiology : official journal of the International Federation of Clinical Neurophysiology, vol. 118, pp. 918-27, Apr. 2007.

[35] M. A. Kramer, E. D. Kolaczyk, and H. E. Kirsch, "Emergent network topology at seizure onset in humans," Epilepsy Research, vol. 79, pp. 173-186, May 2008.

[36] F. Wendling, P. Chauvel, A. Biraben, and F. Bartolomei, "From intracerebral EEG signals to brain connectivity: identification of epileptogenic networks in partial epilepsy.," Frontiers in systems neuroscience, vol. 4, p. 154, Jan. 2010.

[37] J. R. Terry, O. Benjamin, and M. P. Richardson, "Seizure generation: The role of nodes and networks.," Epilepsia, June 2012.

[38] K. Lehnertz and C. Elger, "Can Epileptic Seizures be Predicted? Evidence from Nonlinear Time Series Analysis of Brain Electrical Activity," Physical Review Letters, vol. 80, pp. 5019-5022, June 1998.

[39] F. Mormann, K. Lehnertz, P. David, and C. E. Elger, "Mean phase coherence as a measure for phase synchronization and its application to the EEG of epilepsy patients," Physica D: Nonlinear Phenomena, vol. 144, pp. 358-369, Oct. 2000.

[40] B. Litt and J. Echauz, "Prediction of epileptic seizures," The Lancet Neurology, vol. 1, pp. 22-30, May 2002.

[41] F. Mormann, R. G. Andrzejak, T. Kreuz, C. Rieke, P. David, C. E. Elger, and K. Lehnertz, "Automated detection of a preseizure state based on a decrease in synchronization in intracranial electroencephalogram recordings from epilepsy patients.," Physical review. E, Statistical, nonlinear, and soft matter physics, vol. 67, p. 021912, Feb. 2003.

[42] M. Winterhalder, B. Schelter, T. Maiwald, A. Brandt, A. Schad, A. Schulze-Bonhage, and J. Timmer, "Spatio-temporal patient-individual assessment of synchronization changes for epileptic seizure prediction.," Clinical neurophysiology : official journal of the International Federation of Clinical Neurophysiology, vol. 117, pp. 2399-413, Nov. 2006.

[43] I. Osorio and Y.-C. Lai, "A phase-synchronization and random-matrix based approach to multichannel time-series analysis with application to epilepsy.," Chaos (Woodbury, N.Y.), vol. 21, p. 033108, Sept. 2011.

[44] G. Ouyang, X. Li, Y. Li, and X. Guan, "Application of wavelet-based similarity analysis to epileptic seizures prediction.," Computers in biology and medicine, vol. 37, pp. 430-7, Apr. 2007.

[45] R. G. Andrzejak, D. Chicharro, C. E. Elger, and F. Mormann, "Seizure prediction: any better than chance?," Clinical neurophysiology : official journal of the International Federation of Clinical Neurophysiology, vol. 120, pp. 1465-78, Aug. 2009.

[46] M. S. D. Kerr, S. P. Burns, J. Gale, J. Gonzalez-Martinez, J. Bulacio, and S. V. Sarma, "Multivariate analysis of SEEG signals during seizure.," Proceedings of the Annual International Conference of the IEEE Engineering in Medicine and Biology Society, vol. 2011, pp. 8279-82, Jan. 2011.

[47] S. Santaniello, S. P. Burns, A. J. Golby, J. M. Singer, W. S. Anderson, and S. V. Sarma, "Quickest detection of drugresistant seizures: an optimal control approach.," Epilepsy $\&$ behavior : E\&BB, vol. 22 Suppl 1, pp. S49-60, Dec. 2011.

[48] L. D. Iasemidis, D.-S. Shiau, J. C. Sackellares, P. M. Pardalos, and A. Prasad, "Dynamical resetting of the human brain at epileptic seizures: application of nonlinear dynamics and global optimization techniques.," IEEE transactions on biomedical engineering, vol. 51, pp. 493-506, Mar. 2004.

[49] M. Chávez, M. Le Van Quyen, V. Navarro, M. Baulac, and J. Martinerie, "Spatio-temporal dynamics prior to neocortical seizures: amplitude versus phase couplings.," IEEE transactions on bio-medical engineering, vol. 50, pp. 571-83, May 2003.

[50] M. Palus, V. Komárek, Z. Hrncír, and K. Sterbová, "Synchronization as adjustment of information rates: detection from bivariate time series.," Physical review. E, Statistical, nonlinear, and soft matter physics, vol. 63, p. 046211, Apr. 2001.

[51] M. Eichler, "On the Evaluation of Information Flow in Multivariate Systems by the Directed Transfer Function," Biological Cybernetics, vol. 94, pp. 469-482, Mar. 2006.

[52] D. Y. Takahashi, L. A. Baccalá, and K. Sameshima, "Information theoretic interpretation of frequency domain connectivity measures.," Biological cybernetics, vol. 103, pp. 463-9, Dec. 2010.

[53] L. Barnett, A. B. Barrett, and A. K. Seth, "Granger Causality and Transfer Entropy Are Equivalent for Gaussian Variables," Physical Review Letters, vol. 103, p. 238701, Dec. 2009.

[54] T. Schreiber, "Measuring Information Transfer," Physical Review Letters, vol. 85, p. 461, July 2000.

[55] D. Marinazzo, M. Pellicoro, and S. Stramaglia, "Causal Information Approach to Partial Conditioning in Multivariate Data Sets.," Computational and Mathematical Methods in Medicine, vol. 2012, p. 303601, 2012.

[56] S. Sabesan, L. B. Good, K. S. Tsakalis, A. Spanias, D. M. Treiman, and L. D. Iasemidis, "Information flow and application to epileptogenic focus localization from intracranial EEG.," IEEE transactions on neural systems and rehabilitation engineering, vol. 17, pp. 244-53, June 2009.

[57] C. Stamoulis and B. S. Chang, "Multiscale information for network characterization in epilepsy.," Proceedings of the Annual International Conference of the IEEE Engineering in Medicine and Biology Society, vol. 2011, pp. 5908-11, Jan. 2011.

[58] C. Stamoulis, L. J. Gruber, D. L. Schomer, and B. S. Chang, "High-frequency neuronal network modulations encoded in 
scalp EEG precede the onset of focal seizures.," Epilepsy \& behavior : EESB, vol. 23, pp. 471-80, Apr. 2012.

[59] M. Lindner, R. Vicente, V. Priesemann, and M. Wibral, "TRENTOOL: a Matlab open source toolbox to analyse information flow in time series data with transfer entropy.," BMC neuroscience, vol. 12, p. 119, Jan. 2011.

[60] J. Geweke, "Measurement of Linear Dependence and Feedback Between Multiple Time Series," Journal of the American Statistical Association, vol. 77, pp. 304-313, June 1982.

[61] E. Florin, J. Gross, J. Pfeifer, G. R. Fink, and L. Timmermann, "The effect of filtering on Granger causality based multivariate causality measures.," NeuroImage, vol. 50, pp. 577-88, Apr. 2010.

[62] L. Barnett and A. K. Seth, "Behaviour of Granger causality under filtering: Theoretical invariance and practical application.," Journal of neuroscience methods, vol. 201, pp. 404-419, Oct. 2011.

[63] A. Korzeniewska, M. Maczak, M. Kamiski, K. J. Blinowska, and S. Kasicki, "Determination of information flow direction among brain structures by a modified directed transfer function (dDTF) method.," Journal of neuroscience methods, vol. 125, pp. 195-207, May 2003.

[64] M. Kamiski, "Determination of transmission patterns in multichannel data.," Philosophical transactions of the Royal Society of London. Series B, Biological sciences, vol. 360, pp. 947-52, May 2005.

[65] L. Faes and G. Nollo, "Assessing directional interactions among multiple physiological time series: the role of instantaneous causality.," Proceedings of the Annual International Conference of the IEEE Engineering in Medicine and Biology Society, vol. 2011, pp. 5919-22, Jan. 2011.

[66] K. J. Blinowska, "Review of the methods of determination of directed connectivity from multichannel data.," Medical 83 biological engineering $\& 3$ computing, vol. 49, pp. 521-9, May 2011.

[67] D. Marinazzo, G. Wu, M. Pellicoro, L. Angelini, and S. Stramaglia, "Information Flow in Networks and the Law of Diminishing Marginal Returns: Evidence from Modeling and Human Electroencephalographic Recordings," PLoS ONE, vol. 7, p. e45026, Sept. 2012.

[68] J. X. Tao, M. Baldwin, A. Ray, S. Hawes-Ebersole, and J. S. Ebersole, "The impact of cerebral source area and synchrony on recording scalp electroencephalography ictal patterns.," Epilepsia, vol. 48, pp. 2167-76, Nov. 2007. 